\title{
Postconsummatory delay and goal box confinement ${ }^{1}$
}

PETER J. MIKULKA, JOHN R. VOGEL, AND NORMAN E. SPEAR RUTGERS-THE STATE UNIVERSITY

Four groups of rats were run in a factorial design varying goal box size (large vs small) with a 0 or 30 sec postconsummatory delay. Ss receiving postconsummatory delay during acquisition were more resistant to extinction than nondelayed Ss. As predicted, Ss delayed in the larger goal box were more resistant to extinction than those delayed in the smaller goal box.

The effect of a preconsummatory delay on the acquisition and extinction of an instrumental response is well established (Renner, 1964). However, little attention has been given to the effect of detaining $S$ in the goal situation after the completion of the consummatory response, a postconsummatory delay (PCD). Fehrer (1956), McCain \& Bowen (1967), and Vogel \& Mikulka (1966) have reported that a PCD during acquisition led to increased resistance to extinction. One interpretation of this finding is that during the PCS interval $S$ is repeatedly exposed to an empty food cup and nonreinforcement cues. These nonreinforcement cues become conditioned to the instrumental food cup response and subsequently maintain performance during extinction. Extending this assumption, the more similar the PCD-goal responses are to the instrumental goal response, the stronger this conditioning should be. If during the PCD $S$ is able to leave the food cup area and then reapproach it, this "reapproaching" response would be more similar to the instrumental approach response than that of an $S$ confined at the food cup during the PCD interval. The former condition should be expected to result in a greater resistance to extinction. In support of this, Trapold \& Doren (1966) have shown a facilitated PREE in Ss run $8 \mathrm{in}$. in the goal box to an empty food cup as compared to Ss simply placed at the empty food cup.

The present experiment combined the use of small and large goal boxes with a PCD. It was assumed that the large, relatively open goal box would increase the similarity of the food cup approach response to the instrumental running response, whereas the smaller goal box would minimize the similarity of these responses.

Method

The design was a 2 by 2 factorial in which goal box size (large vs small) and PCD (0 vs $30 \mathrm{sec)}$ were combined orthogonally. The Ss were 28 experimentally naive male albino rats of the Sprague-Dawley strain, approximately 60 days old at the start of the experiment. The apparatus was the same as that described by Vogel, Mikulka, \& Spear (1966, Experiment 1) with the addition of interchangeable goal boxes: $12 \times 12 \times$
8 in. high (large) and $12 \times 3.5 \times 5$ in. high (small). All Ss were handled for six days prior to the start of the experiment. On Days 5 and 6 each $S$ was allowed to explore the start and alley segments of the apparatus for $3 \mathrm{~min}$. Fifty acquisition trials were given at five trials per day with an intertrial interval of $15 \mathrm{sec}$. The reward used was one $\mathbf{0 4 5} \mathrm{g}$ Noyes pellet. Twenty extinction trials followed in which all Ss were confined for $15 \mathrm{sec}$ in their respective goal boxes.

\section{Results and Discussion}

Acquisition. Analyses of variance were performed on the last 20 trials of acquisition. There was a tendency for the $30 \mathrm{sec}$ PCD Ss to run slower over all three speed measures. However, this effect was only significant in the goal measure $(F=24.19, \mathrm{df}=1 / 24$, $p<.01)$. The marked depression in speeds at the goal began after 25-30 acquisition trials and continued throughout the remainder of acquisition (see Fig. 1). This depression in the response measure nearest the goal concurs with earlier research conducted in our laboratory (Vogel \& Mikulka, 1966). PCD-nonreinforcement cues can be expected to generalize backwards along the instrumental approach chain and appear most strongly at the goal, as was the case here. Further, the finding of greatest depression nearest the goal is

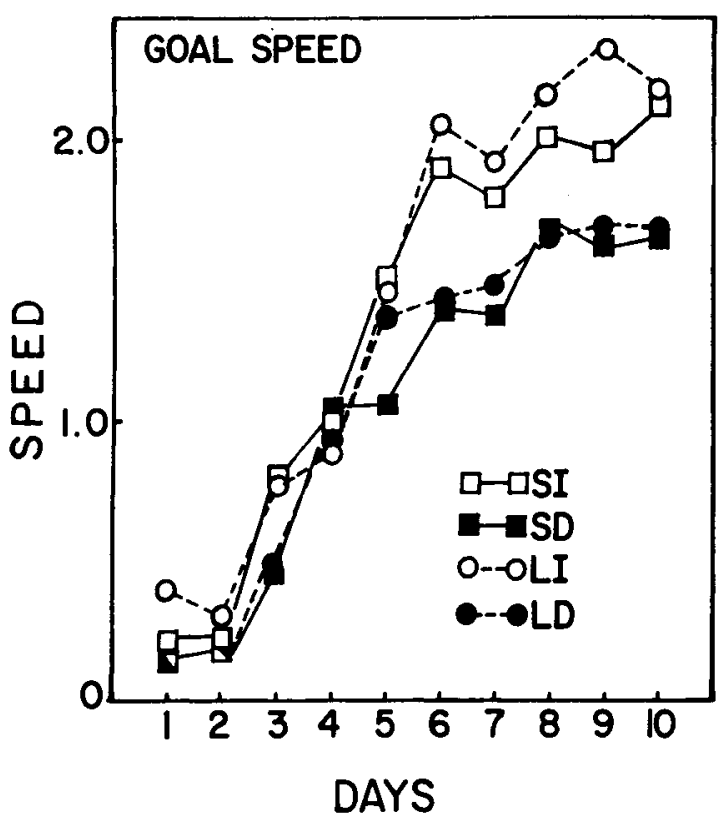

Fig. 1. Acquisition goal speed with large (L) or small (S) goal boxes and a post consummatory delay (D) or immediate removal (I). 


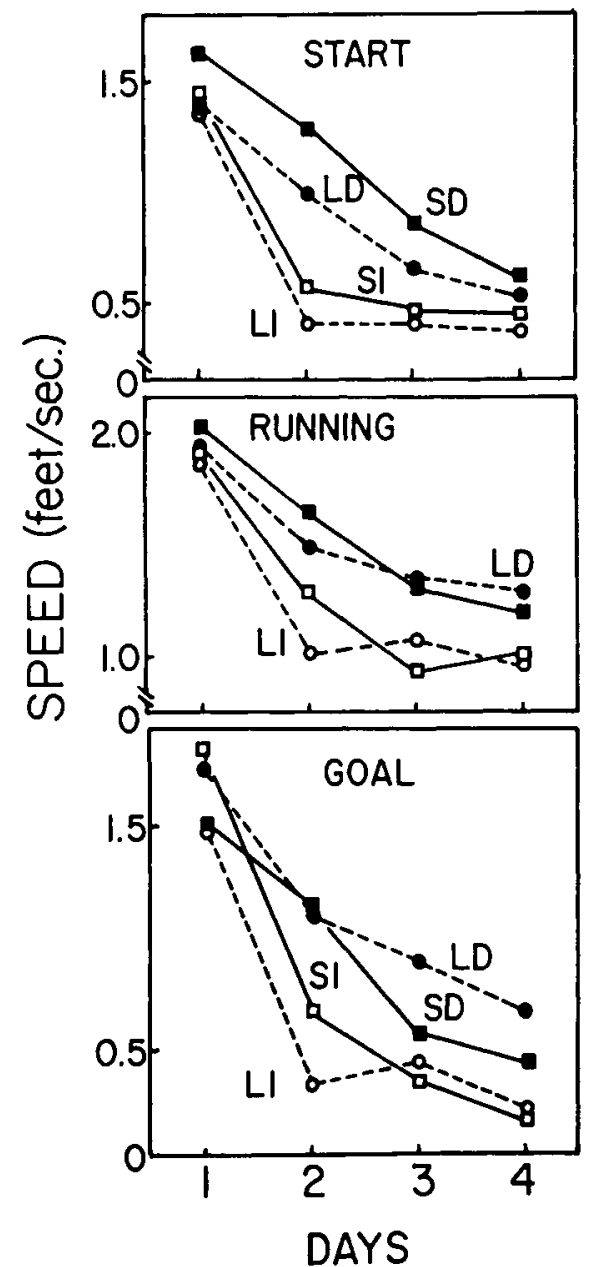

Fig. 2. Start, running, and goal speeds during extinction.

also consistent with partial reinforcement data (Spence, 1960, chap. 6). There was no significant effect of goal box size on any speed measure, nor did it interact with the PCD variable.

Extinction. Analyses of extinction indicated that Ss receiving a $30 \mathrm{sec} \mathrm{PCD}$ in acquisition ran faster throughout extinction than the immediate Ss. This effect was significant in start, running, and goal speeds $(F=7.50,7.15,17.73$, respectively, $\mathrm{df}=1 / 24$, $\mathrm{p}<.01)$. This supports earlier findings of an enhanced resistance to extinction following PCD training. Again, the apparent similarity of these data to those for partial reinforcement supports the interpretation of PCD in terms of the conditioning of nonreinforcement cues to the instrumental response. Such an interpretation is consistent with Capaldi (1966) and Amsel (1962).
Analyses of the Goal Box Size by PCD interaction yielded no significant effect in start or running speeds, but was significant in goal speed $(F=5.16, d f=1 / 24, p$ $<.05)$. The Ss that received a $30 \mathrm{sec}$ PCD in conjunction with the large goal box were more resistant to extinction than those Ss given their PCD in the small goal box (see Fig. 2). This effect fits with the acquisition finding of greatest sensitivity to PCD nearest the goal and suggests the existence of a goal gradient for nonreinforcement effects. Simply, the association of the nonreinforcement cues should be the strongest at the goal, and should be expected to have a greater effect on the maintenance of extinction responding at the goal. The present findings are taken as tentative support for the hypothesis that PCD cues will condition more strongly to the instrumental sequence if the PCD-goal responses are more similar to the instrumental approach response.

An alternative prediction was possible, based on aversion to confinement during the PCD. This would predict an increased resistance to extinction following PCD training in the smaller goal box. This clearly was not the case. Therefore, it is reasonable to suggest that the specific goal box activity following consumption of the reward is important in affecting subsequent resistance to extinction.

\section{References}

AMSEL, A. Frustrative nonreward in partial reinforcement and discrimination learning: some recent history and a theoretical extension. Psychol Rev., 1962, 49, 306-328.

CAPALDI, E. J., \& POYNOR, H. Aftereffects and delay of reward. J. exp. Psychol, 1966, 71, 80-88

FEHRER, E. Effects of amount of reinforcement and of pre- and postreinforcement delays on learning and extinction. J. exp. Psychol., 1956, 52, 167-176.

McCAIN, G., \& BOWEN, J. Pre-and postreinforcement delay with a small number of acquisition trials. Psychon, Sci, 1967, 7, 121-2.

RENNER, K. E. Delay of reinforcement: a historical review. Psychol. Bull. 1964, 61, 341-361.

SPENCE, K. W. Behavior theory and learning. Englewood Cliffs: Prentice Hall, 1960.

TRAPOLD, M. A., \& DOREN, D. G. Effect of noncontingent partial reinforcement on the resistance to extinction of a nunway response. J. exp. Psychol, 1966, 71, 429-431.

VOGEL, J. R., \& MIKULKA, P. J. An examination of the effects of postconsummatory delay and reward magnitude on runway performance in the rat. Paper presented at the East. Psychol. Assoc. 1966.

VOGEL, J., MIKULKA, P., \& SPEAR, N. E. Effect of interpolated extinction and level of training on the "depression effect." $J$. exp. Psychol, 1966, 72, 51-60.

\section{Note}

1. This research was supported by USPHS Grant MH-08888-01 and Rutgers University Research Council Grant No. 685. 